\title{
Optical Distance Sensors
}

\section{Arihant Siyal ${ }^{1}$}

NIOS A-24/25, Institutional Area, Sector - 62, Gautam Budh Nagar, NOIDA - 201 309, Uttar Pradesh, India

Abstract: The article deals with three optical distance sensors, which are mounted on an apparatus so that they could be tested, compared \& analysed. This study can be a helping hand in future for the people who want to acquire any particular detail or information on any one of these sensors. This research work adds another information about tested sensor for anyone trying to get hands on information for the three sensors or their applications.

Keywords: sensor; calibration; gauge, measurement.

\section{Introduction}

Optical distance sensors are the result of decades of opto-electronics research \& innovation. This branch of photonics deals with the use of electronic devices that track, produce and modulate light in order to perform an incredibly diverse range of functions. Light can be used to measure the distance of a sensor to a barrier or substrate in the scope of distance measurement. Optoelectronic instruments are associated with the permeability of sub-visible ionizing radiation (gamma, X-rays, etc.), although in the visible and near-visible electromagnetic spectrum, optical distance sensors typically harness light. Amongst these various optical distance sensing concepts, either a laser or a light-emitting diode (LED) is most widely used to produce an amplified light beam that is used for distance measurement when an object senses reflection. As a function of the Time-of-Flight (ToF) concept, these modulated beams are used to measure distance [1-6].

\section{Description of tested sensors}

The analogue distance sensor SHARP GP2YOA41SKOF (Fig. 1) has a detection range of $1.5^{\prime \prime}$ to 12 " $(4 \mathrm{~cm}$ to $30 \mathrm{~cm})$. The narrower range gives you better resolution readings, and this sensor is better for detecting very nearby particles because of the substantially lower detection distance. The analogue voltage represents the distance, making this sensor very simple to use. A perfect way to add obstacle avoidance or motion sensing to your robot or some other project is the SHARP GP2YOA21 distance sensor. This sensor is very simple to use, with a detection range of 4" to 32 " $(10 \mathrm{~cm}$ to $80 \mathrm{~cm})$ and an analogue voltage indicating the size. This famous IR based long range $(20-150 \mathrm{~cm})$ sensor from SHARP electronics is capable of generating analogue signals according to the distance from the target object. By connecting them to an ADC port, they can be easily interfaced with SPDuino or other micro controllers.

IR Sensors operate to detect a selected light wavelength in the Infra-Red (IR) spectrum by using a particular light sensor. You will look at the strength of the received light by using an LED that emits light at the same wavelength as what the sensor is looking for.
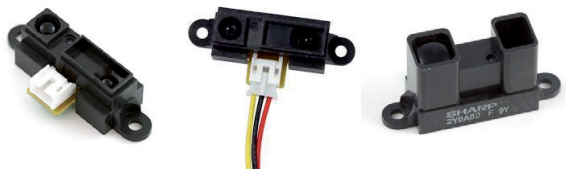

Figure 1: Sensor Sharp 0A41SK; sensor Sharp 2Y0A21; sensor Sharp 2Y0A02. 


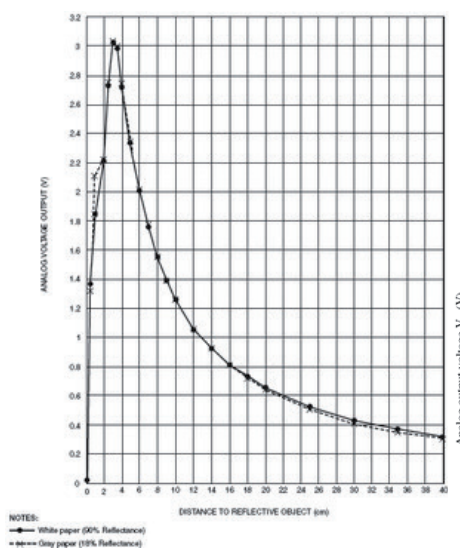

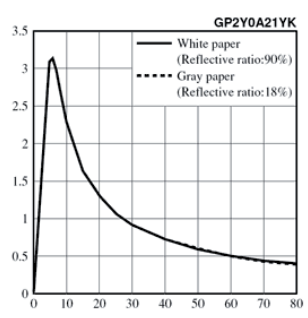

Distance to reflective object $\mathrm{L}(\mathrm{cm})$

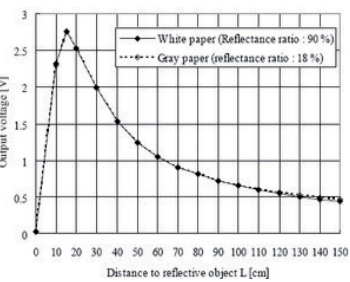

Figure 2: Voltage Distance Graph (SHARP 0A41SK; 2Y0A21; 2Y0A02).

Pulse oximeter sensor - The sensor module contains the MAX30102 sensor (Fig. 2) and other accessories. The MAX30102 module is an integrated oximeter and pulse sensor $[6,7]$.

\section{Apparatus for experiments}

The apparatus (Fig. 3) is based on a wooden block with a sliding rack mounted on top which helps in sliding the holder on which subjects could be slapped on so they could stay still or if needed the holder can be loosened up to take readings while subject is tilted.

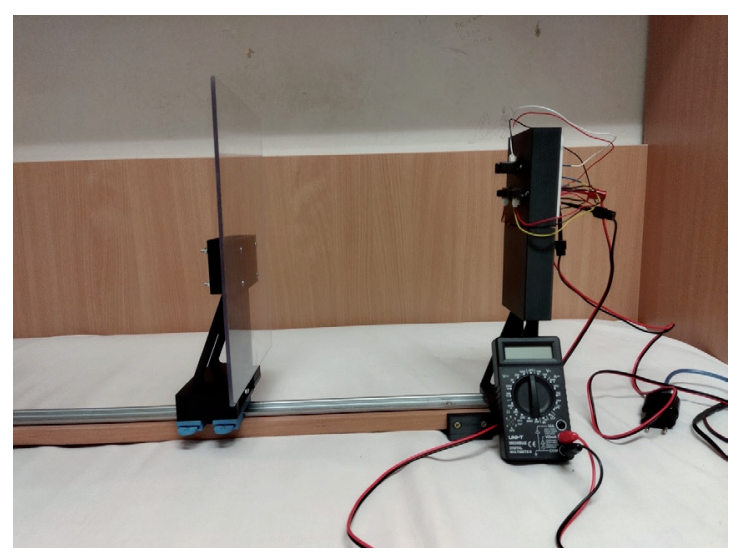

Figure 3: Test apparatus for optical sensors.

The apparatus (Fig. 3) is connected to DC power which turns on the sensors, we have slot for each sensor so that the multimeter can be connected to particular sensor to derive the readings. Once we have all of this connected we use measured plastic blocks to create the desired distance between the subject holder and the apparatus. Plastic etalon blocks were manufactured and verified with a maximum deviation of $0.05 \mathrm{~mm}$. Further, we put up material on the subject holder (Coloured paper, Transparent objects, mirrors, glasses) and measure output from the sensor due to a subject to verify the experiment as per say or the datasheet provided by the manufacturer. The three sensors (Fig. 4) are mounted on a block; Sharp 2Y0A02 (Top), Sharp 2Y0A21 (Bottom right), Sharp 2Y041SK (Bottom left). Breadboard (Fig. 4) is being used to ease the connections and power all the three sensors at the same time.

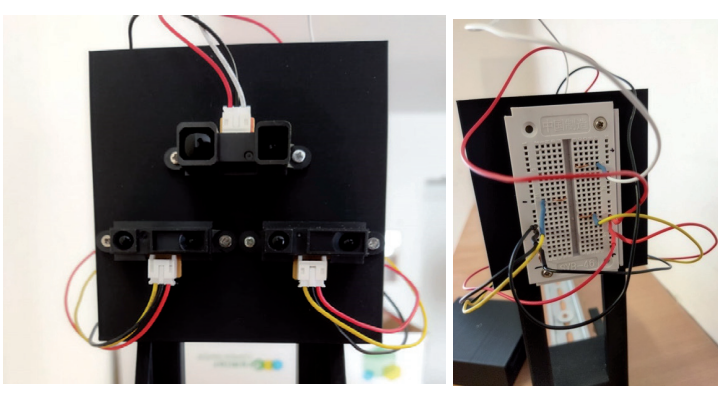

Figure 4: Sensors and solderless breadboard mounted on a removable block.
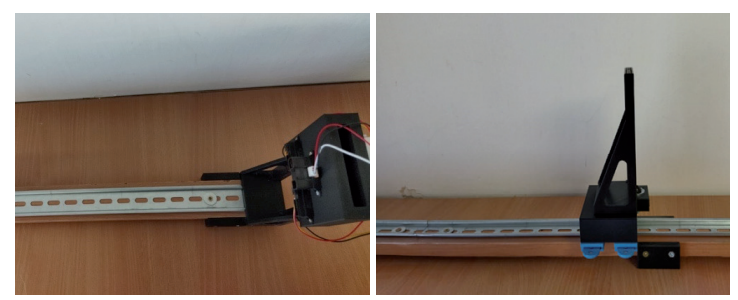

Figure 5: DIN rail sliding system. 
The multimeter was used to measure the variating values from sensors reacting to different materials. Stell DIN rail is as a base of the apparatus and a significant part of the experiment which allows us to verify the properties of the sensor by letting the subjects slide on (Fig. 5). A plastic removable block with sensors are mounted onto the rack which is slidable making it easy to move the subject on DIN rail. Plastic etalon blocks were made as didactic tool instead of original steel parallel length gauges (Fig. 6). The distance between the sensors and the reflection surface of the subject was gradually adjusted using these plastic standard blocks over the entire range of distance measurement using the sensors. The measured subject with the reflecting surface was mounted on a sliding system (Fig. 7) by means of a plug-in system, which allows a quick exchange of the subject. It is possible to attach different types of reflecting surfaces with different properties to the subject (colour, surface roughness, reflectivity, etc.).
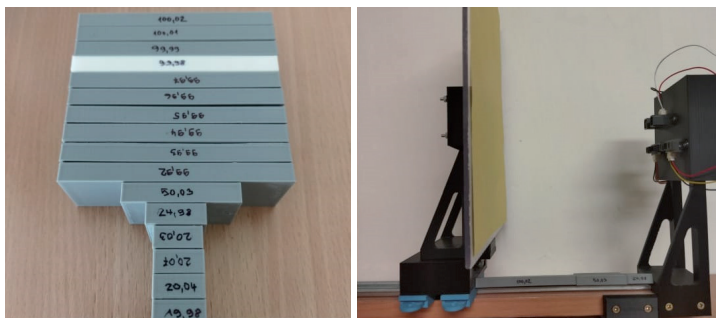

Figure 6: Setting the distance between the sensors and reflection surface using the plastic etalon block.

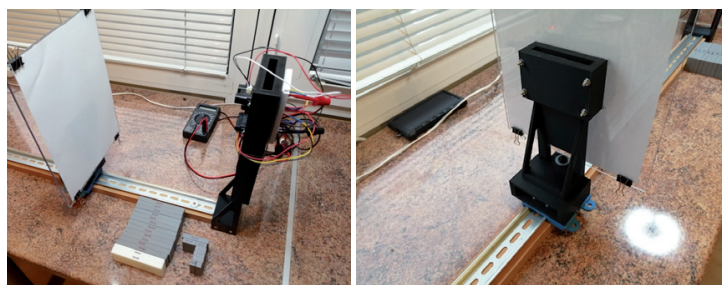

Figure 7: Measured subject with the reflecting surface was mounted on a sliding system.

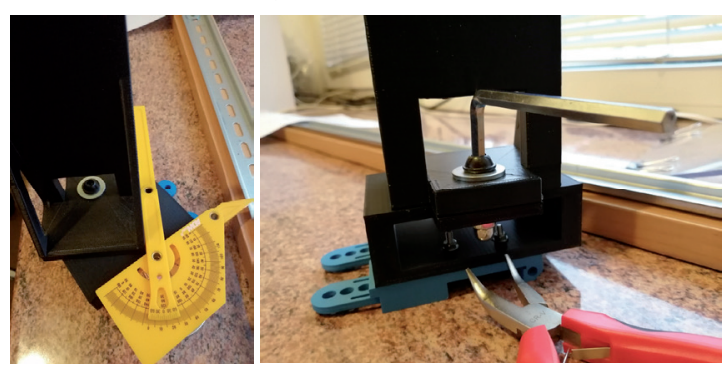

Figure 8: Rotation of reflecting surface.
In addition, the sliding system includes a rotating joint, which also allows the subject's reflecting surface to rotate relative to the sensors being tested. This apparatus also allows testing of sensors with the subject's reflected reflection surface. The rotation of the reflecting surface is adjusted by means of a pantograph protractor and then fixed by means of a fixing screw.

\section{Results of experiments}

Multimeter used in the experiment has its own uncertainty and error, Manufacturer has defined a particular relationship for the uncertainty: DC voltage: $200 \mathrm{mV} ; \pm(0.25 \%+2) / 2000 \mathrm{mV} / 20 \mathrm{~V} /$ $200 \mathrm{~V} / 1000 \mathrm{~V} ; \pm(0.5 \%+2)$

Even though all three sensors were being used at the same time no disturbance or hindrance in the performance of either was experienced throughout the experiment. It was not possible to verify it on paper, but physically when the readings were drawn from the sensors simultaneously it was clear that the sensors were not interfering each other's performance.

Repeatability and measurement range of sensors are evaluated in first experiment. The experiment consisted of 18 readings and repeated exercise with all the three sensors which started at a distance of $1174.83 \mathrm{~mm}(117.48 \mathrm{~cm})$ from the sensors with the subject holder carrying different materials and just to get a scope of the diversity of the experiment 198 readings were drawn from each sensor. Maximum length of the plastic block ranged up to $100.02 \mathrm{~mm}$ $(10 \mathrm{~cm})$ and minimum up till $10.00 \mathrm{~mm}(1 \mathrm{~cm})$. Various colours (RAL-3024, RAL-6027, RAL-6018, RAL1016) of reflective surface have been tested (Fig. 9).

Sensors differ in behaviour depending on distance which is one of the main reasons what sets these sensors apart from each other. For e.g., SHARP 2Y041SK the shortest-range sensor reacts to most of the subjects alarmingly at a distance varying between 20-40mm; SHARP 2YOA21 the mid-range sensor responds more between $60-80 \mathrm{~mm}$; SHARP 2Y0A02 the long ranger is most sensitive between 100-125 mm. The truth being that colour of the material really did not make a significant difference in the readings from the sensors, some of them did push the sensor readings to fluctuate at a high rate but did not put a noticeable dent to the overall experiment, the most used material was paper with nine different and divergent colour (Fig. 10). The 


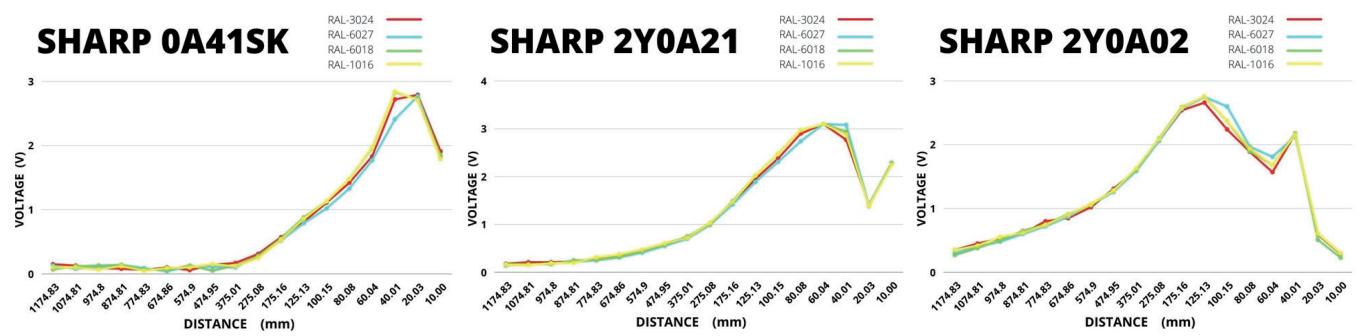

Figure 9: Repeatability of tested sensors.
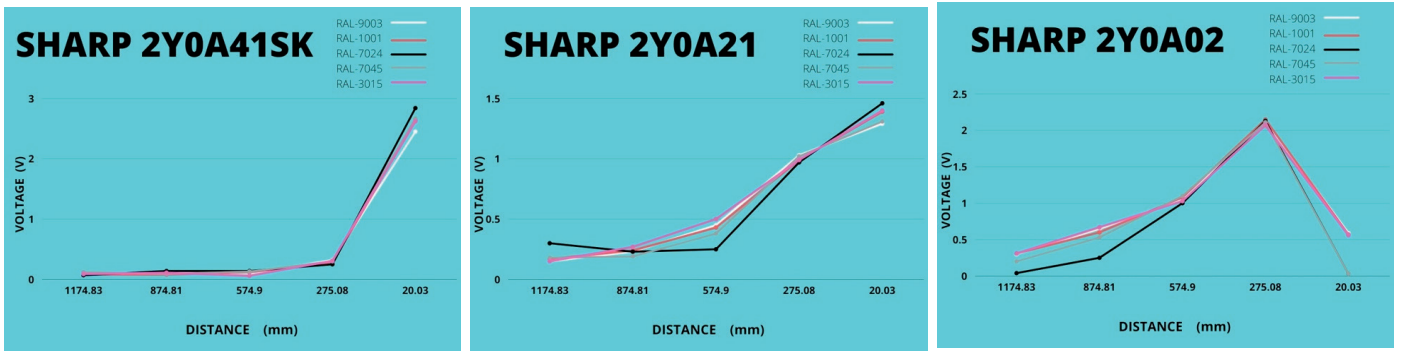

Figure 10: Dependence on colour and material.

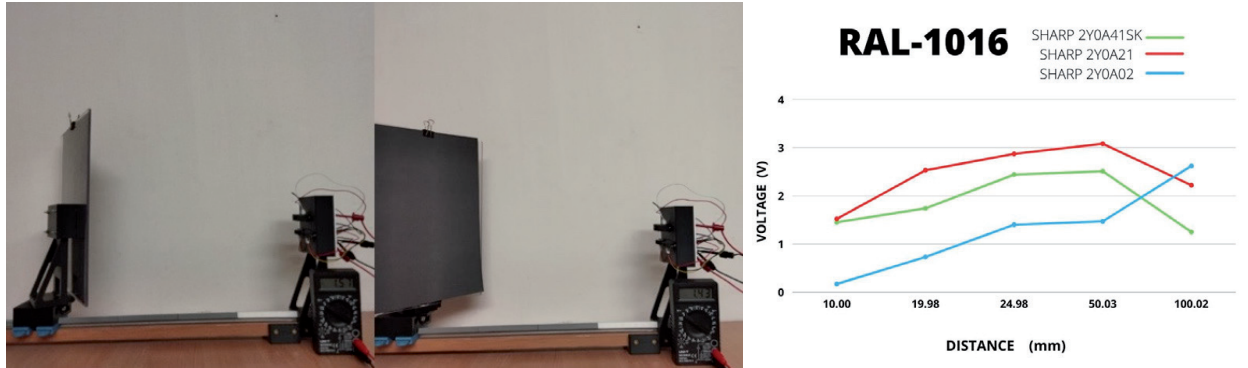

Figure 11: Tilted subject with reflective surface and sensors response for RAL-1016 color.
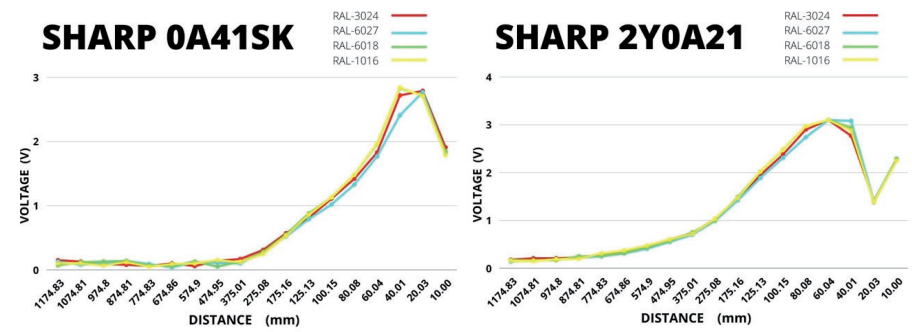

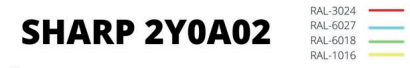

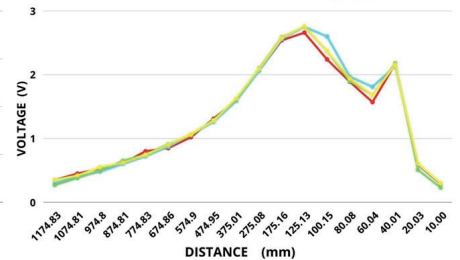

Figure 12: Sensors response for tilted subject with mirrored and transparent reflective surface.

tested sensors have been used with various colours RAL-9003 - Signal White; RAL-1001 - Beige; RAL-7024 - Graphite Grey; RAL-7045 - Telegray 1; RAL-3015 Light Pink; RAL-1016 - Sulphur Yellow; RAL-6018 Yellow Green; RAL-3024 - Luminous Red; RAL-6027 - Light Green.

When the subjects were tilted, we could see a variation in the readings mostly when the tilt was more towards left and the subject was coloured paper. While using transparent board the readings tanked down due to the tilt, On the other hand when mirror was used the readings spiked. The use of a transparent and mirrored surface caused an unreliable measurement, which also follows from the principle of operation of the sensors (Fig. 12).

\section{Conclusions}

The sensors are phenomenal and while experimenting with them it was clear that they have a lot of potential and can be used in several interesting places, for example PLC, cup dispenser, end of the line. A circuit can be compiled which can 
consist of a microcontroller (Arduino), sensors, LCD screen, buzzer, pushbuttons and the project can be used to measure distance of a particular object.

This research aimed at determining the capabilities of the three given optical distance sensors and put together all the possible information by experimenting with these sensors which could be a helping hand for anyone who decides to use these small but very powerful sensors.

During the time span of this research, it was a pleasurable opportunity to explore the apparatus and while exploring it with coloured paper the sensor did not react much differently to various colours but the reaction of sensors to transparent board was a sight or the mirror, the sensors performed very well even during the daylight. Tilted subject didn't let the sensors capabilities down but rather pushed them to the edge and gave an example of the performance limits. The two short range sensors namely Sharp 2Y0A41SK, Sharp 2Y0A21 were seen struggling with the subject at a far distance. On the other hand, the long-range sensor Sharp 2Y0A02 performed rather well even at long or short range. Even working together at any given point, the sensors did not hinder each other's performance or showed any signs of incumbency.

\section{References and Notes}

1. Šimčák, F., Kelemen, M., Virgala, I., \& Miková, L’. (2013). Optical Line Recognition Sensors for Line Follower. Acta Mechanica Slov, 17(4), 16-21. doi: 10.21496/ams.2013.041.

2. Jakubkovič, E., Kelemenová, T., \& Koláriková, I. (2021). Displacement Measurement in the Vertical Axis of the Measuring Microscope using Laser Triangulation Sensor. Acta Mechanica Slov., 25(2), 14-18. doi: 10.21496/ams.2021.019.

3. Palanisamy, A. (2020). Home Automation Using PLC and Arduino. Acta Mechanica Slov., 24(1), 56-68. doi: 10.21496/ ams.2020.021.

4. Juruš, O., \& Ferenčík, P. (2021). Ultrasonic Sensors used to Detect the Position of the Mobile Service Robot. Acta Mechanica Slov., 25(1), 28-32. doi: 10.21496/ams.2021.010.

5. Gmiterko, A., Kelemen, M., Virgala, I., \& Miková, L’. (2014). Educational Model of Four Legged Robot. Acta Mechanica Slov., 18(3-4), 68-73. doi: 10.21496/ams.2014.032.

6. Virgala, I., Miková, L., \& Kelemen, M. (2018). Robotic snakes. Acta Mechanica Slo.v, 22(3), 38-43. doi: 10.21496/ ams.2018.025.

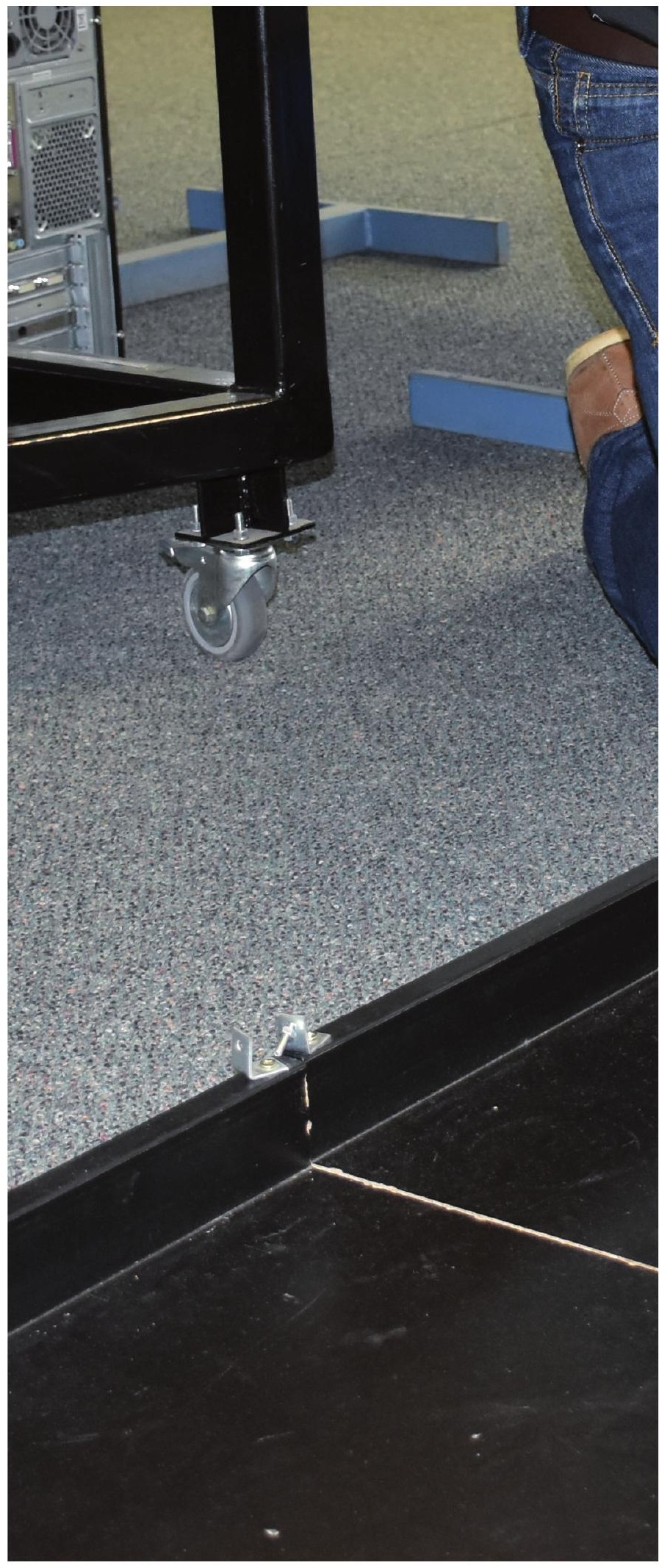

